Metalle können auf diese Weise nicht eruiert werden. Man kann sie nicht anders feststellen, als durch Zerstören der Hefe, wobei sich jedes Metall verwandelt und in sein Exkrement übergeführt wird, als welches es dann erkannt werden kann. Die Hefe wird zu diesem Zweck mit Essig oder Ätzwasser versetzt; den eingetrockneten Rückstand kann man auf metallische Exkremente untersuchen. Findet man Rost darauf, ist das ein Zeichen für Vorhandensein von Eisen, Grünspan ein solches von Kupfer. Man darf aber nicht jede grüne Farbe für Grünspan ansprechen; sie kann auch von grünem Bolus oder Rost herrühren. Wird solche grüne Hefe in destillierten Essig gelegt, wird Bolus noch grüner, Rost aber schwarz.

Die Galläpfelprobe kennt Falloppio, wenn auch nicht ganz zutreffend, da er auch Alaun damit nachweisen will.

Auf Geruch und Geschmack soll man sich nicht verlassen, da es sich meist um Gemische handelt, die ein sicheres Urteil nicht zulassen.

Mag auch so ziemlich alles in dieser Untersuchungsanweisung heute überholt sein, handelt es sich doch um einen ersten, hochwichtigen Anfang qualitativer Untersuchung von Mineralwasser, der erst lange nachher überholt wurde. Wieviele solche Untersuchungen Geßner noch selber durchführen konnte, weiß man nicht. Da er selber sehr fleißig destillierte, schon aus pharmazeutischen Gründen, wie man aus seinen Briefen weiß, ist es durchaus möglich, daß er umfangreichere Untersuchungen gemacht hat; auf keinen Fall hat er sich nur literarisch dafür interessiert. Eine zweite Ausgabe seines Bäderbuches hätte wahrscheinlich allerlei Zusätze gebracht. Man wird aber schon jetzt nicht bestreiten können, daß Geßner mit Recht auch der Name eines Vaters der schweizerischen Balneologie gebührt.

Bernhard Milt.

\title{
Descartes und Theophrast von Eresos
}

In seiner berühmten, zuerst anonym im Jahre 1637 erschieneners Schrift: «Discours de la méthode pour bien conduire sa raison et chercher la vérité dans les sciences» hat Descartes der Physik die sicheren mathematischen Grundlagen geschaffen. Im ersten Teil seiner Abhandlung legt er die Gründe dar, welche ihn veranlaßt haben, eine neue Methode wis. 
senschaftlicher Forschung zu suchen. Er tut dies (1637, S. 10, Z. 7 von oben, 1668 S. 9) mit folgenden Worten: "Je ne dirai rien de la Philosophie, sinon que voyant qu'elle a été cultivée par les plus excellens esprits qui ayent vécu depuis plusieurs siècles, et que néanmoins il ne s'y trouve encore aucune chose dont on ne dispute, et par conséquent qui ne soit douteuse, je n'avais point assez de présomption pour espérer d'y rencontrer mieux que les autres. Et que considérant combien il peut y avoir de diverses opinions touchant une même matière, qui soient soutenues par des gens doctes, sans qu'il y en puisse avoir jamais plus d'une seule qui soit vraie, je reputais presque pour faux tout ce qui n'était que vraysemblable.

Puis pour les autres sciences, d'autant qu'elles empruntent leurs principes de la Philosophie, je jugeais qu'on ne pouvait avoir rien bâti qui fût solide, sur des fondements si peu fermes.»

Diese Stelle, auf die ich durch Prof. Gonseths Vortrag über «Die Dialektisierung des Erkennens» (1943) aufmerksam wurde, in welchem ihr letzter Abschnitt zitiert ist, kam mir in ihrem allgemeinen Gedankengang und in einzelnen ihrer Ausdrücke merkwürdig bekannt vor. War ich doch solchen Ausführungen auch schon begegnet, aber weder bei Descartes, noch bei einem andern Gelehrten der neueren Zeit, wohl aber beim Aristoteles-Schüler Theophrast von Eresos (370-285 a. Chr.). Dieser legt nämlich im I. Buch seiner Causae plantarum Kap. 21 \$ und 4 dar, daß als Ursache der Fruchtreife und der Entwicklung der Pflanzen die Wirkung des Klimas und der Sonne, sowie die Konstitution jeder Pflanze betrachtet werden müsse, «ob sich nun die Konstitution durch großen oder geringen Saftgehalt... und dergleichen auszeichnet oder durch ihre Wärme oder ihre Kälte; denn auch letztere Eigenschaften gehören zur Konstitution (\$4). Von diesen sind die zuerst erwähnten sozusagen alle durch die sinnliche Wahrnehmung erkennbar, das Warme und das Kalte dagegen wird, weil es der Wahrnehmung nicht zugänglich ist, sondern nur dem logischen Denken, immer wieder in Zweifel gezogen und zum Gegenstand von Kontroversen gemacht, wie alle andern Dinge, welche nur mit Hilfe des logischen Denkens beurteilt werden. Über diese Begriffe (d. h. des Warmen und des Kalten) irgendwie Klarheit zu schaffen, ist aber deshalb notwendig, weil viele Vorgänge der Natur auf diese beiden Prinzipien zurückgeführt werden. Nun müssen solche Vorgänge insgesamt auf Grund der Begleiterscheinungen untersucht werden. Auf Grund der Be- 
gleiterscheinungen beurteilen wir nämlich die wirkenden Kräfte und bilden uns über sie unsere Anschauung.»

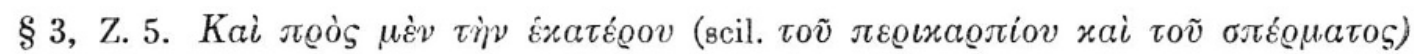

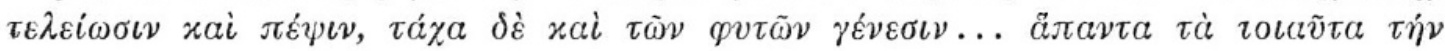

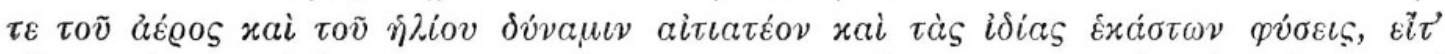

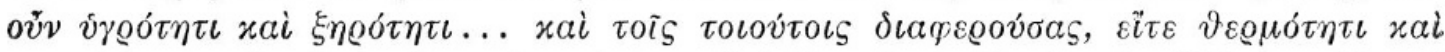

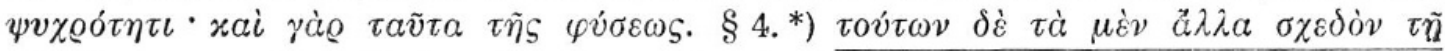

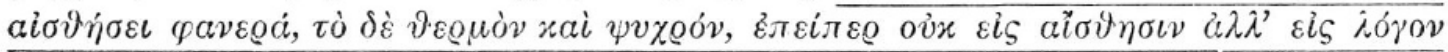

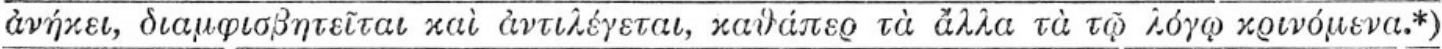

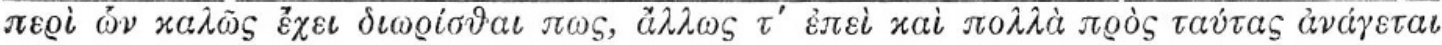

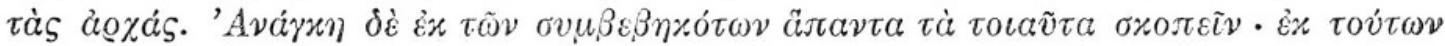

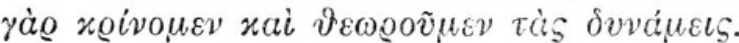

Ein Vergleich dieses Textes mit demjenigen von Descartes ergibt zunächst eine auffallende Ưbereinstimmung im allgemeinen Gedankengang. So spricht aus beiden Zitaten die Unzufriedenheit mit den Ergebnissen, welche die Philosophie (bei Descartes), resp. das logische, rein verstandes. mäßige Denken (bei Theophrast) geliefert haben. Diese innere Not hat beide Forscher dazu veranlaßt, eine neue Methode zu suchen, welche die Gewähr bietet, daß mit ihr die Wahrheit gefunden werden kann.

Neben dieser prinzipiellen Übereinstimmung in den Gedankengängen beider Forscher bestehen aber noch zwei Vergleichspunkte spezieller Natur. So hat Descartes' Äußerung, daß sich in der Philosophie nichts findet, über das nicht disputiert, gestritten werde, eine auffallende Parallele in Theophrasts Feststellung, daß den mit Hilfe des Logos gefundenen

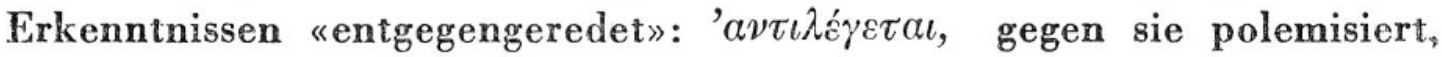
über sie diskutiert werde.

Sodann entspricht dem Descartes'schen «aucune chose ... qui ne soit douteuse» das theophrastische $\delta \iota \alpha \mu \varphi \iota \sigma \beta \eta \tau \varepsilon i \tau \alpha \iota:$ :die Ansichten gehen darüber weit auseinander, es wird als völlig unentschieden, unsicher, zweifelhaft angesehen».

Neben dieser grundsätzlichen besteht noch die vielleicht mehr zufällige Übereinstimmung, daß Descartes im Anschluß an seine theoretischen Ausführungen als praktisches Beispiel den Blutkreislauf im Anschluß an Harvey bespricht und als Ursache der Bewegung die hohe Temperatur des Herzens betrachtet, während Theophrast seine neue Methode dazu verwendet, den warmen oder kalten Charakter der Pflanzen festzustellen.

* Übersetzung Theodors von Gaza: \& 4 quarum rerum caetera prope sensui plane occurrunt. Calor autem et frigiditas, quia non sensu, sed ratione percipi possint, nimirum dubitantur atque in crebra oblocutione versantur: scilicet quemadmodum caetera quae ratione solummodo dijudicare valemus. 
Daß diese Versuche beide Forscher zu unrichtigen Schlüssen geführt haben, mag als Ironie des Schicksals bezeichnet werden, ändert aber nichts an der Tatsache, daß die durch den einen wie den andern gefundenen neuen Methoden die Wissenschaft in hohem Maße gefördert haben.

Angesichts dieser auffallenden Übereinstimmung erhebt sich die Frage, ob Descartes selbständig zu seiner Einstellung gegenüber der zeitgenössischen Forschungsmethode gelangt sei, oder in Kenntnis von Theophrasts Ausführungen. Seine völlige Selbständigkeit wäre durchaus denkbar. Befand er sich doch dem damaligen scholastischen Wissenschaftsbetrieb gegenüber in derselben Situation wie Theophrast gegenüber der von Aristoteles angewandten Methode, von welcher die Scholastik nichts anderes als die Weiterentwicklung bildete, nachdem die Kirche dank den Bemühungen Alberts des Großen (1193-1280) das Studium der aristotelischen Philosophie erlaubt und in der Folge deren Resultate weitgehend anerkannt hatte. Demnach sind es dieselben Gründe gewesen, welche Descartes veranlaßt haben, die scholastische Methode zu verlassen, welche auch Theophrast bewogen haben, sich von der Methode seines Meisters abzukehren und sie durch eine bessere zu ersetzen. Die Duplizität des Falles ließe sich somit sehr wohl mit Hilfe der Identität der äußeren Bedingungen erklären, welche beide Forscher zu denselben Überlegungen geführt haben.

Nun stellten wir aber auf S. 18 fest, daß, außer der Übereinstimmung im allgemeinen Gedankengang, in den Äußerungen beider Forscher noch eine spezielle Ähnlichkeit besteht. Beide betonen nämlich die Unsicherheit der Resultate (chose douteuse $=\delta \iota(\alpha \mu \iota \sigma \beta \eta \tau \varepsilon \tau \tau \alpha \iota)$ und die daraus resultierende

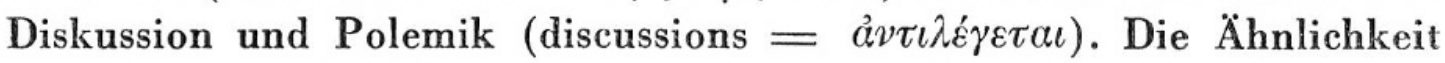
von Descartes Ausführungen mit denjenigen Theophrasts ist besonders groß, wenn man nicht den griechischen Text, sondern die lateinische Übersetzung des Theodor von Gaza in Betracht zieht. Wenn nämlich Descartes das $\delta \iota \alpha \mu \varphi \iota \sigma \beta \eta \tau \varepsilon \tilde{\tau} \tau \alpha \iota$ des Theophrast mit «chose douteuse» wiedergibt, so entspricht dies dem «nimirum dubitantur» Theodors von Gaza genau. Die Übersetzung «dubitantur» ist zwar richtig, aber nicht die einzig mögliche. Bedeutet doch das griechische Verb zunächst: «über diese Dinge gehen die Ansichten weit auseinander", sie sind darum zweifelhaft. Daß Descartes' Ausdruck mit demjenigen Theodors übereinstimmt, spricht dafür, daß Descartes nicht den griechischen Urtext, son- 
dern die lateinische Übersetzung gelesen hat. Allerdings zitiert er den Theophrast weder im Discours noch in seinen übrigen Schriften (siehe General-Register der Ausgabe Adam-Tannery 1913). Das scheint mir aber kein Beweis gegen seine Bekanntschaft mit Theophrasts Abhandlungen über die Causae zu sein. Die erwähnte Übereinstimmung in Einzelheiten der Ausdrucksweise, sowie die Tatsache, daß beide Forscher die Anwendbarkeit ihrer neuen Methode am Beispiel der Körperwärme - Descartes beim Menschen, Theophrast bei der Pflanze - erweisen wollen, läßt sich ungezwungen damit erklären, daß Descartes von den Gedankengängen des Theophrast Kenntnis gehabt hat. Das ist durchaus möglich, weil Theophrasts Schriften vor dem Erscheinen von Descartes' Discours öfters im griechischen Urtext und noch öfter in der lateinischen Übersetzung des Theodor von Gaza verlegt worden sind. Zudem versichert ja Descartes (1637 S. 6, Z. 6 v. unten, 1668, S. 6, Z. 13 v. oben), er habe alle Bücher gelesen, die besonders interessante Dinge behandeln. ${ }^{1}$ Man könnte sich allerdings wundern, daß er als Physiker und Mathematiker auch botanische Schriften studiert habe. Demgegenüber ist aber darauf hinzuweisen, daß Theophrasts Ausführungen über seine Methode nicht in seinen Historiae plantarum, sondern in seinen Causae plantarum enthalten sind. Dieser Titel nun, welcher die Causae hervorhebt, kann Descartes sehr wohl veranlaßt haben, das Buch zu lesen, weil auch in seinem Discours die «causes» eine große Rolle spielen. Ich glaube darum zur Annahme berechtigt zu sein, daß die Übereinstimmung von Descartes' Ausführungen mit der erwähnten Theophrast-Stelle nicht in das Gebiet der Duplizität der Fälle eingeordnet werden muß, sondern darauf beruht, daß Descartes die Causae plantarum des Theophrast gekannt hat. Ob ihm erst bei deren Lektüre Zweifel an der Brauchbarkeit der scholastischen Methode aufgestiegen sind, oder ob ihn Theophrast nur in seinen eigenen Zweifeln bestärkt hat, oder endlich, ob er bei ihm nur den adaequaten Ausdruck seiner Zweifel gefunden hat, läßt sich wohl kaum entscheiden. $\mathrm{Da} \beta$ er aber in der Formulierung von ihm abhängig ist, dafür liefern die vorstehenden Ausführungen den bündigen Beweis. Beide For. scher, Descartes, der Franzose aus der Zeit des dreißigjährigen Krieges, und Theophrast, der Zeitgenosse Alexanders des Großen, dürfen darum als einander geistesverwandt bezeichnet werden. Theophrasts Zweifel an der Zuverlässigkeit der rein gedanklichen Konstruktion ist somit nicht,

1 J'avais parcouru tous les livres, traitans de celles qu'on estime les plus curieuses et les plus rares, qui avaient pû tomber entre mes mains. 
wie bis vor kurzem angenommen werden mußte (vgl. Senn 1933 S. 200), bisher übersehen worden. Vielmehr scheint ihn schon Descartes entdeckt und in seiner Bedeutung erkannt zu haben. Jedenfalls müssen die grundsätzlichen Änderungen, welche beide Forscher durch ihre Zweifel an der Zuverlässigkeit der aristotelisch-scholastischen Methode veranlaßt haben, als Wendepunkte in der Geschichte der Naturforschung betrachtet werden.

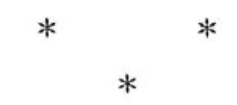

Während aber Theophrast und Descartes in der negativen Einstellung gegenüber dem Aristotelismus resp. der Scholastik einig waren, gingen sie bei der Überwindung dieser Denkweise, also bei ihren positiven Bemühungen, völlig verschiedene Wege. Während Theophrast die Beobachtung der Naturvorgänge und ihrer Begleiterscheinungen als Grundlage der Naturerkenntnis proklamierte, letztere also a posteriori mit Hilfe der Sinneswahrnehmung zu gewinnen suchte, zweifelte Descartes an der Zuverlässigkeit der durch die Sinne gewonnenen Erkenntnisse und basierte seine Methode a priori auf wenige Praemissen, auf denen er nicht nur die Geometrie, welcher diese Methode durchaus adaequat ist, sondern sein gesamtes Weltbild mit Hilfe seiner «raison bien conduite» aufbaute. Diese ist aber nichts anderes als der Logos des griechischen Philosophen Parmenides (516/14-451/49 a. Chr.). Nun hat aber Theophrast die mit dem Logos gewonnenen Erkenntnisse für unsicher erklärt. Zwischen ihm und Descartes besteht somit in dieser Beziehung ein diametraler Gegensatz.

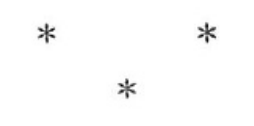

Daß Theophrast mit seiner neuen Methode Erfolg gehabt hat, ergibt sich aus der Tatsache, daß die Leuchten unter den antiken Naturforschern und Ärzten, wie Straton, Herophilos, Erasistratos und Soranos, sich ihrer bedient haben. Beim Niedergang der Naturwissenschaften gegen das Ende der Antike ist diese rein naturwissenschaftliche Methode verloren gegangen. Aber auch Descartes' aprioristische Methode fand bei einer großen Gruppe von Wissenschaftern, besonders bei Mathematikern, Anklang. Doch bald erhoben sich auch gegnerische Stimmen, und zwar nicht nur von Seite der Kirche und speziell der Jesuiten, auf welche Descartes aus äußeren Gründen Rücksicht nehmen mußte, sondern auch von Naturforschern, welche auf Grund ihrer Beobachtungen und Experimente 
mehrere der mit Descartes' aprioristischer Methode gewonnenen Resultate als irrig erwiesen hatten (Boorsch 1937 S. 176). Obwohl die von Descartes' Gegnern vertretene Richtung in der Naturforschung die Oberhand gewann, indem sie Beobachtung und Experiment in zunehmendem Maße anwandte und auf diese Weise bis auf den heutigen Tag große Erfolge erzielte, muß auch die Publikation von Descartes' Discours als ein Wendepunkt in der Geschichte der Naturforschung bezeichnet werden, dies aber nicht wegen der positiven, sondern wegen der negativen Seite seiner Methode, d. h. wegen des Mißtrauens, das Descartes den Sinneswahrnehmungen gegenüber hegte. Dieses Mißtrauen nötigte auch seine Gegner, ihre Beobachtungen in Natur und Experiment viel kritischer zu beurteilen und auf ihre Tatsächlichkeit exakter zu prüfen, als dies v o r Descartes der Fall gewesen war. Dank also der negativen Seite von dessen Methode gelangten die auf Beobachtung fußenden Forscher zu gesicherten Resultaten, d.h. zur «Wahrheit», die auch Descartes mit heißem.Bemühen gesucht hatte. Darum muß ihm ein großes Verdienst um die Entwicklung der Naturwissenschaften in der neueren Zeit zuerkannt werden, so wie es sich Theophrast von Eresos für die Naturforschung der Antike erworben hatte. Insofern nun, als beide durch den Zweifel an der überkommenen Forschungsmethode angeregt worden sind, eine neue Methode zu ersinnen, dürfen ihre Namen trotz der grundsätzlichen Verschiedenheit der von ihnen gefundenen Methoden neben einander genannt werden, wie dies im Titel dieses Aufsatzes geschehen ist.

\section{Literatur}

1937 Boorsch, J. Etat présent des études sur Descartes. Etudes françaises, Société d'Edition «Les Belles Lettres», Paris.

Descartes, René. Discours de la Méthode pour bien conduire sa rạison, et chercher la vérité dans les sciences.

1637 - Anonym erschienen bei Jan Maire in Leyden.

1668 - Unter Descartes Namen erschienen bei Charles Angot, Paris.

1943 Gonseth, F. Die Dialektisierung der Erkenntnis. Eidgenössische Technische Hochschule, Kultur- und staatswissenschaftliche Schriften Nr. 33. Polygraphischer Verlag A.-G. Zürich.

1933 Senn, G. Die Entwicklung der biologischen Forschungsmethode in der Antike und ihre grundsätzliche Förderung durch Theophrast von Eresos. Veröffentlichungen der Schweiz. Gesellschaft f. Geschichte der Medizin und der Naturwissenschaften. H. R. Sauerländer \& Co., Aarau.

Theophrast von Eresos, Causae plantarum, lateinische Übersetzung des Theodor von Gaza, Lugduni, G. Rouilly, 1552.

G. Senn. 